\title{
A constitutive law for degrading bioresorbable polymers
}

Hassan Samami and Jingzhe Pan

Department of Engineering, University of Leicester, Leicester, UK

\begin{abstract}
This paper presents a constitutive law that predicts the changes in elastic moduli, Poisson's ratio and ultimate tensile strength of bioresorbable polymers due to biodegradation. During biodegradation, long polymer chains are cleaved by hydrolysis reaction. For semicystalline polymers, the chain scissions also lead to crystallisation. Treating each scission as a cavity and each new crystal as a solid inclusion, a degrading semi-crystalline polymer can be modelled as a continuum solid containing randomly distributed cavities and crystal inclusions. The effective elastic properties of a degrading polymer are calculated using existing theories for such solid and the tensile strength of the degrading polymer is predicted using scaling relations that were developed for porous materials. The theoretical model for elastic properties and the scaling law for strength form a complete constitutive relation for the degrading polymers. It is shown that the constitutive law can capture the trend of the experimental data in the literature for a range of biodegradable polymers fairly well.
\end{abstract}

Key words: effective moduli, cavity inclusion, crystal inclusion, crystalline and amorphous polymers, multi-phase material.

\section{Introduction}

There is a worldwide attempt to develop and use bioresorbable medical implants such as coronary stents and fixation screws made of bioresorbable polymers. The bioresorbable screws and plates have been used for internal fixation in patients for a number of years. The bioresorbable stents are currently being used in several countries. Unlike permanent implants made of stainless steels or titanium alloys, bioresorbable implants "disappear" after serving their functions in the human body. When designing bioresorbable implants, it is important to understand how the mechanical properties of the polymer materials change as they degrade. Degradation of bioresorbable polymers occurs by uptake of water and attack of water molecules to ester bonds of the polymer chains in a time dependent process known as hydrolysis degradation (Buchanan et al., 2008). The basic "damage" mechanism is polymer chain scission caused by the hydrolysis reaction. These chain scissions lead to significant reduction in the mechanical property of the polymers (Ashby and Jones, 2005, Tsuji, 2000, Tsuji, 2002, Tsuji, 2003, Tsuji and Del Carpio, 2003, Tsuji and Muramatsu, 2001). Some 
typical bioresorbable polymers are semi-crystalline. New nano-sized crystals can be formed as the consequence of the extra mobility of the polymer chains due to chain scission. The increase in crystallinity can be as high as 40\% (Bouapao et al., 2009, Saha and Tsuji, 2006, Tsuji et al., 2004). Because the crystals have a higher Young's modulus than the amorphous phase, the new crystals act as an enhancement phase that increases the Young's modulus of the semi-crystalline polymer. Despite a polymer chain cleavage is an event at the atomistic scale, each chain-scission can be treated as an effective spherical cavity in the polymer. A new crystal on the other hand can be treated as an inclusion of different Young's modulus. It is then possible to calculate the effective moduli of a degrading polymer for continuum solid containing these cavities and inclusions. A representative volume element (RVE) for the polymer is considered and it is assumed that the size of the cavities and crystals remains constant, but their numbers increase as degradation proceeds. Gleadall (2014) performed such calculations using finite element analysis. However the numerical calculations could only provide empirical fitting equations for the Young's modulus. These fitting functions are unnecessarily dependent on the details of the finite element models. Furthermore Gleadall did not consider the full constitutive response and ignored Poisson ratio and strength of degrading polymers which are important if one wishes to use the constitutive law for device design. This paper presents a complete and analytical constitutive law for degrading polymers.

Numerous studies have been carried out for the effective moduli of composite materials. The Voigt approximation is probably the simplest model which assumes the strain throughout a composite is uniform and equal to the average strain. The Reuss approximation assumes that the stress throughout a composite is uniform and equal to the average stress. Hill proved that the Voigt approximation and Reuss approximation are actually the upper and lower bounds of the effective elastic moduli (Hill, 1963). Hashin and Shtrikman also provided bounds for the elastic moduli and tensors of isotropic composites reinforced by aligned continuous fibres or randomly positioned particles which are similar to Walpole bounds but better than the Voigt and Reuss bounds (Hashin and Shtrikman, 1962, Walpole, 1966). The effective moduli estimated by Eshelby using the EIM method is only valid in the limit of low porosity (dilute limit) (Eshelby, 1957). The EIM yields the same result as the dilute approximation which ignores the interaction between reinforcing particles. The self-consistent schemes (SCS) use material properties of a composite for infinite medium, i.e. the inclusion phase is assumed to see an effective medium of unknown properties. Hill presented the overall constraint tensor 
for an isotropic continuum containing a spherical cavity based on the self-consistent method. He assumed that inclusions are spheres distributed in a way such that the composite is statistically isotropic overall (Hill, 1963). Mackenzie (1950) was probably the first who calculated the elastic constants of solid containing circular cavities. His scheme, known as the generalised self-consistent scheme (GSCS), assumes that particles are surrounded by a concentric shell of known property embedded in an effective medium of unknown properties (Mackenzie, 1950). Buiansky proposed a method to avoid the problem of giving meaningless values for the effective moduli of a composite material which arises when the volume fraction of inhomogeneities is increased (Budiansky, 1965). The SCS is a two-phase model while GSCS is a three-phase model which according to Aboudi yields a better result (Aboudi, 1991). Hashin also proposed a composite spheres model which derives the bulk modulus of a composite material composed of a collection of spheres each of which consists of a spherical core (particle) and a concentric spherical shell (matrix) (Hashin, 1962). The result of the composite spheres model coincides with that of GSCS. The overall moduli of a two-phase elastic composite material can also be determined by differential scheme (DS). In this method the composite material is constructed explicitly from an initial material through a series of incremental additions. To account for phase interaction effects, the Mori-Tanaka's scheme (MTS) relates the average stress or average strain tensors of matrix and inhomogeneities (inclusion) phases by the fourth order concentration tensors (Mori and Tanaka, 1973). All of the SCS, GSCS, DS and MTS schemes that are typically derived based on the concept of representative volume element (RVE) are approximate schemes of interacting defects (Kachanov et al., 1994). The RVE is a sub-volume of sufficient size of an inhomogeneous medium. Since the material is assumed to be statistically homogeneous, the mechanical properties of the entire composite material are assumed to be the same as those of the RVE.

This paper is structured as following: section 2 presents a model for predicting the effective moduli of degrading crystalline polymers. The model of is also reduced to predict the effective moduli of amorphous polymer. The effective moduli of several amorphous and crystalline polymers are taken from the literature and fitted with the model in sections 3 and 4. Section 5 shows our calculation of the Poisson's ratio for the same polymers considered in sections 3 and 4 . The tensile strength of these polymers is fitted using a scaling relation in section 6. 


\section{A mathematical model for prediction of constitutive behaviour of degrading bioresorbable polymers}

Treating each chain-scission as an effective cavity, a degrading amorphous polymer can be modelled by a continuum solid containing randomly distributed cavities with a common radius. Fig. 1 shows a degrading polymer whose molecular chains are broken by hydrolysis reactions. It is assumed that each chain scission leads to a cavity in the polymer matrix.

A semi-crystalline polymer undergoes an increased crystallinity during the hydrolysis degradation (Tsuji et al., 2004). Treating each crystal as a solid inclusion and each chain scission as a cavity, a degrading semi-crystalline polymer can be modelled by a continuum solid containing cavities and solid inclusions. A crystal has a higher stiffness than the amorphous matrix while a cavity is a region whose stiffness is zero.

Fig. 2 shows our model for a degrading semi-crystalline polymer before and after degradation. In the solid mechanics literature, the cavities and inclusions can be referred to as inhomogeneity together. Fig.3 shows a representative volume element (RVE) of the solid polymer subjected to uniformly remote stress $\sigma^{0}$ that contains an inhomogeneity $\Omega^{(1)}$ with elastic stiffness $C_{I}^{(1)}$ (which is zero) and an inhomogeneity $\Omega^{(2)}$ whose elastic stiffness is $C_{I}^{(2)}$ . Since the size of the particles is much smaller compared to the size of the RVE, it can be assumed with they don't interact with the boundaries of the RVE.

The average stress $\bar{\sigma}$ of a 3-phase material with perfect bonding between constituents shown as Fig. 3 can be obtained by (Aboudi, 1991, Mura, 1987).

$$
\bar{\sigma}=f_{M} \bar{\sigma}_{M}+f_{I}^{(1)} \bar{\sigma}_{I}^{(1)}+f_{I}^{(2)} \bar{\sigma}_{I}^{(2)}
$$

where $f_{M}$ and $f_{I}^{(\alpha)}$ are the volume fractions of the matrix and inhomogeneities, respectively. Similarly, $\bar{\sigma}_{M}$ and $\bar{\sigma}_{I}^{(\alpha)}$ are the average stress of the matrix and inhomogeneities $(\alpha=1,2)$, respectively.

According to the average stress theorem when a uniform stress $\sigma^{0}$ is applied on the boundary of this solid material, the average stress of the material is equal to $\sigma^{0}$. Thus, Eq. (1) becomes 


$$
\sigma^{0}=f_{M} \bar{\sigma}_{M}+f_{I}^{(1)} \bar{\sigma}_{I}^{(1)}+f_{I}^{(2)} \bar{\sigma}_{I}^{(2)}
$$

Using Hook's law Eq. (2) becomes

$$
C \varepsilon^{0}=f_{M} C_{M} \bar{\varepsilon}_{M}+f_{I}^{(1)} C_{I}^{(1)} \bar{\varepsilon}_{I}^{(1)}+f^{(2)} C_{I}^{(2)} \bar{\varepsilon}_{I}^{(2)}
$$

Where $C_{M}$ and $\bar{\varepsilon}_{M}$ are the elastic stiffness and the average strain of the matrix phase. The elastic stiffness of a cavity inclusion is $C_{I}^{(1)}=0$, Therefore, Eq. (3) reduces to

$$
C \varepsilon^{0}=f_{M} C_{M} \bar{\varepsilon}_{M}+f^{(2)} C_{I}^{(2)} \bar{\varepsilon}_{I}^{(2)}
$$

From the analysis of the average strain we obtain

$$
\bar{\varepsilon}=f_{M} \bar{\varepsilon}_{M}+f_{I}^{(1)} \bar{\varepsilon}_{I}^{(1)}+f_{I}^{(2)} \bar{\varepsilon}_{I}^{(2)}
$$

According to the average strain theorem $\bar{\varepsilon}=\varepsilon^{0}$ (Mura, 1987), we have

$$
f_{M} \bar{\varepsilon}_{M}=\varepsilon^{0}-f_{I}^{(1)} \bar{\varepsilon}_{I}^{(1)}-f_{I}^{(2)} \bar{\varepsilon}_{I}^{(2)}
$$

Substitution of Eq. (6) into Eq. (4) gives the effective stiffness of degrading bioresorbable polymers as

$$
C=C_{M}-C_{M} f_{I}^{(1)}\left(\bar{\varepsilon}_{I}^{(1)} / \varepsilon^{0}\right)+\left(C_{I}^{(2)}-C_{M}\right) f_{I}^{(2)}\left(\bar{\varepsilon}_{I}^{(2)} / \varepsilon^{0}\right)
$$

In tensor notation form it can be written as

$$
C_{i j k l}=C_{M_{i j k l}}-C_{M_{i j k l}} f_{I}^{(1)}\left(\bar{\varepsilon}_{I_{k l}}^{(1)} / \varepsilon_{k l}^{0}\right)+\left(C_{I_{i j k l}}^{(2)}-C_{M_{i j k l}}\right) f_{I}^{(2)}\left(\bar{\varepsilon}_{I_{k l}}^{(2)} / \varepsilon_{k l}^{0}\right)
$$

This will be referred to as Model One in our following discussions, which is actually constitutive law of effective moduli of a three phase particulate composite material when $C_{I_{i j k l}}^{(1)}=0$. It implies that the effective moduli of semi-crystalline bioresorbable polymers can be determined from the elastic moduli of crystalline phase provided the average strain $\bar{\varepsilon}_{I_{k l}}^{(\alpha)}(\alpha=1,2)$ in the phases are known.

The bulk and shear moduli when RVE is subjected to remote hydrostatic pressure $\varepsilon_{k k}^{0}$ and shear strain $\varepsilon_{12}^{0}$ are given by

$$
K=K_{M}-K_{M} f_{I}^{(1)}\left(\bar{\varepsilon}_{I_{k k}}^{(1)} / \varepsilon_{k k}^{0}\right)+\left(K_{I}^{(2)}-K_{M}\right) f_{I}^{(2)}\left(\bar{\varepsilon}_{I_{k k}}^{(2)} / \varepsilon_{k k}^{0}\right)
$$




$$
\mu=\mu_{M}-\mu_{M} f_{I}^{(1)}\left(\bar{\varepsilon}_{I_{12}}^{(1)} / \varepsilon_{12}^{0}\right)+\left(\mu_{I}^{(2)}-\mu_{M}\right) f_{I}^{(2)}\left(\bar{\varepsilon}_{I_{12}}^{(2)} / \varepsilon_{12}^{0}\right)
$$

The concentration factor $\bar{\varepsilon}_{I_{i j}}^{(\alpha)} / \varepsilon_{i j}^{0}$ can be determined from micro-mechanical schemes such as MTS and EIM that can be found in Table 1.

The Poisson's ratio and the Young modulus are given by

$$
\begin{gathered}
E=9 K \mu /(3 K+\mu) \\
v=3 K-2 \mu / 2(3 K+\mu)
\end{gathered}
$$

There are other schemes for the concentration factor in the literature. The reason to select MTS and EIM models in this study is because EIM is valid for dilute limit and MTS also accounts for the interaction between inclusions. Therefore, the result of Eq. (8) can be compared with the both interacting and non-interacting inclusions.

The constitutive law for amorphous degrading polymer can be obtained from Eq. (8) by setting $f_{I}^{(2)}=0$ as

$$
C_{i j k l}=C_{M_{i j k l}}-C_{M_{i j k l}} f_{I}^{(1)}\left(\bar{\varepsilon}_{I_{k l}}^{(1)} / \varepsilon_{0_{k l}}\right)
$$

The bulk and shear moduli of the amorphous polymer are given by

$$
\begin{aligned}
& K=K_{M}-K_{M} f_{I}^{(1)}\left(\bar{\varepsilon}_{I_{k k}}^{(1)} / \varepsilon_{0_{k k}}\right) \\
& \mu=\mu_{M}-\mu_{M} f_{I}^{(1)}\left(\bar{\varepsilon}_{I_{12}}^{(1)} / \varepsilon_{0_{12}}\right)
\end{aligned}
$$

which is simply the constitutive law for the effective moduli of a two-phase composite material when elastic moduli of the particles (phase 2) within the matrix phase is zero (Aboudi, 1991, Mura, 1987). This will be referred to as Model Two in the following discussion.

\section{Predicting effective moduli of degrading amorphous polymers}

The effective moduli of a degrading amorphous polymer can be calculated from Eq. (13) using the initial conditions and the current average molecular weight of a degrading polymer. The volume fraction of the cavities (porosity) $\left(1 / \mathrm{m}^{3}\right)$ is given by

$$
f_{I}^{(1)}=\sum 4 \pi r^{3} R_{s}
$$


where $R_{s}\left(\mathrm{~mol} \mathrm{~m}^{-3}\right)$ is the number of chain-scissions, $r(\mathrm{~nm})$ is the radius of the cavities. The radius $r$ is the only fitting parameter in the model and unique to each polymer. The number of chain-scissions, $R_{s}$, can be obtained from the molecular weight using a relation developed by Pan and co-workers (Pan, 2014).

$$
\begin{gathered}
\bar{M}_{n}=\frac{M_{n}}{M_{n 0}}=\frac{1-\alpha \bar{R}_{s}^{\beta}}{1+\frac{C_{e 0}}{N_{\text {chain } 0}}\left(\bar{R}_{s}-\frac{\alpha}{m} \bar{R}_{s}^{\beta}\right)} \\
\bar{R}_{s}=R_{s} / C_{e 0}, N_{\text {chain } 0}=\frac{\rho_{0}}{M_{n 0}}
\end{gathered}
$$

where $\bar{M}_{n}$ is the number averaged molecular weight normalised by its initial value, $M_{n 0}$ is the initial number average molecular weight $(\mathrm{g} / \mathrm{mol}), C_{e 0}$ is the initial concentration of ester bonds $\left(\mathrm{mol} / \mathrm{m}^{3}\right), \alpha$ and $\beta$ are empirical parameters for oligomer production that depends on the end or the random scissions (dimensionless), $\rho_{0}$ is initial polymer density $\left(\mathrm{g} / \mathrm{m}^{3}\right), m$ is the degree of polymerization of the oligomers (dimensionless and typically set as 4 to 6 ), $R_{S}$ is the total number of chain scissions per unit volume $\left(\mathrm{mol} / \mathrm{m}^{3}\right)$ and $\bar{R}_{S}$ is the total number of chain scission normalised by the initial number of ester bond per unit volume. The molecular weight can be either calculated using the polymer degradation model developed by Pan and co-workers (Pan, 2014), or taken from experimental data in the literature which is what we chose to do in the current paper. This eliminates the intermediate step of using the degradation model since the purpose here to validate the constitutive law.

Table 2 shows the characteristics of four amorphous films made of poly(L-lactide) (PLLA), poly(D-lactide) (PDLA) and poly(DL-lactide) (PDLLA) respectively that were used by Tsuji etc al (Tsuji, 2002) in their degradation and mechanical experiments. All the initially amorphous PLA films with $18 \times 30 \times 0.05 \mathrm{~mm}^{3}$ size were synthesized to have similar molecular weight and to remain totally amorphous during 24 months hydrolysis degradation. 
Table 2 also provides the radius of the effective cavities for each PLA films using which the Young modulus of the PLA films are fitted using the MTS model.

The porosities of the PLA films of Table 2 as function of degradation time are obtained from Eqs. (16) and (17) which are shown in Fig.4. It shows that the amorphous PDLLA polymer has a higher porosity compared to other amorphous polymers.

Fig.5 shows the results of using Model Two for predicting the Young modulus of the PLA films of Table 2. It shows that the model can capture the general trend of the experimental data fairly well. Using average of the difference between normalised experimental and predicted Young's modulus data defined as Average $\left|\left(E_{\text {exp. }}-E_{\text {Model }}\right) / E_{0}\right|$ gives a level of agreement of 0.07, 0.05, 0.09 and 0.13 between experimental and predicted Young's modulus of the PLLA, PDLA, PDLLA and PDLA/PLLA films, respectively.

\section{Predicting Young's moduli of degrading semi-crystalline polymers}

The effective moduli of the semi-crystalline crystalline polymers can be predicted from Eq. (8). Similar to amorphous polymers the volume fraction of cavities is calculated from Eq. (16). The volume fraction of the crystals can be obtained by using either the crystallisation model (Gleadall et al., 2012, Han and Pan, 2008) or taking directly from experimental measurements. Again we chose the latter since our aim here is to validate the constitutive law. The number of chain scissions of a semi-crystalline polymer, $R_{s}$, can be related to the molecular weight and crystallinity using an equation proposed by Pan and co-workers (Pan, 2014).

$$
\begin{gathered}
\bar{M}_{n}=\frac{M_{n}}{M_{n 0}}=\frac{1+\bar{\omega} X_{c 0}-\alpha \bar{R}_{s}^{\beta}}{\left(1+\bar{\omega} X_{c 0}\right)\left[1+\frac{C_{e 0}}{N_{\text {chain } 0}}\left(\bar{R}_{s}-\frac{\alpha}{m} \bar{R}_{s}^{\beta}\right)\right]} \\
N_{\text {chain } 0}=\frac{\rho}{M_{n 0}}, \bar{\omega}=\frac{\omega}{C_{e 0}} \approx \frac{1}{1-X_{c 0}}
\end{gathered}
$$


where $X_{c 0}$ is the initial degree of crystallinity, $\omega$ is the number of the ester units of crystalline phase per unit volume, $N_{\text {chain } 0}$ is the initial number of molecular chains per unit volume including crystalline phase. Other symbols were introduced in section 3.

Error! Reference source not found. shows characteristics of different semi-crystalline polymers used in different studies by various researchers. The first nine cases were taken from the work by Tsuji and his co-workers (Tsuji and Ikada, 2000, Tsuji et al., 2000). These were in two separate hydrolysis degradation studies in $10 \mathrm{ml}$ phosphate-buffered solutions over a period of 3 years. The case 10 shows a PLLA polymer used by Weir et al (Weir et al., 2004) who used rod specimens for an in vitro hydrolytic degradation study over a period of 44 weeks. The PLLA polymers shown in cases 11-12 were also used as rod samples with 3 and $2 \mathrm{~mm}$ diameter, respectively, by Duek et al (Duek et al., 1999) for an vitro hydrolytic degradation study over a period of 20 weeks.

Error! Reference source not found. also gives the radii of the cavities for each of the PLLA films that were used to fit the constitutive law with the experimental data. It can be seen that the PLLA films with higher crystallinity requires higher radius for the cavity. This agrees with experimental observation that polymers with higher crystallinity absorb more water and degrades faster (Tsuji and Ikada, 2000, Tsuji et al., 2000).

Fig. 6 shows the scission induced crystallinity (experimental data from the literature) and the calculated porosity of polymer cases 1-13 of Table 3 as functions of degradation time.

Fig. 7 shows the fitting between the Model One and the experimental data for the first five semi-crystalline PLLA films of Table 3. It can be observed from this figure that the model can capture the trend of the experimental data fairly well, particularly for the first two years of degradation during which the polymer loses $65 \%$ of its elastic property.

In fact, using the average of the difference between normalized experimental and predicted Young's modulus data shows that the level of agreement between experimental and predicted Young's modulus data is $0.11,0.16,0.19,0.09$ and 0.07 for the polymer cases $1-5$ of Table 3 .

Fig. 8 shows the fitting of the experimental data for Young modulus of the semi-crystalline PLLA films of cases 6-9 of Table 3. It can be observed from this figure that the model can again capture the general trend of the experimental Young modulus particularly for the semicrystalline PLLA cases 6 and 8 shown in Figs. 8 (a) and (c). However, it cannot predict the 
modulus of the polymer case 9 for the last stage of degradation as shown in Fig. 8 (d). The level of agreement between experimental and predicted Young's modulus of the polymer cases 6-9 of Table 3 obtained from the average of difference between normalized experimental and predicted Young's modulus data is given as as 0.11, 0.17, 0.09 and 0.15, respectively.

Fig. 9 again shows that the Model One can successfully capture the general trend of the experimental Young modulus data of the semi-crystalline PLLA films given as cases 10-13 in Table 3. And, the level of agreement between experimental and predicted data of the polymer cases 10-13 is obtained from the average of difference between normalized experimental and predicted Young's modulus data as $0.11,0.14,0.19$ and 0.2 , respectively.

So far, only MTS concentration factors which account for the interaction effects between inclusions has been used in Model One to fit the experimental data of the semi-crystalline polymers. Using EIM in Model One, the predicted Young modulus of, for instance, semicrystalline polymer cases 6-9 is shown as Fig. 10. It can be observed from this figure that the EIM is not good in prediction of the Young modulus of semi-crystalline polymers, as it does not account for the interaction effects between inclusions. Predicting Poisson's ratio of degrading Polymers

The Poisson's ratio of a homogeneous isotropic linear elastic material can be obtained from Eq. (12) using bulk and shear moduli defined as Eq. (9) and Eq. (10) for amorphous polymers and Eq. (14) and Eq. (15) for semi-crystalline polymers. Crystals and amorphous matrix are same polymers of different densities. This study assumes that the Poisson's ratio of the matrix and crystal phases is equal to $v=0.35$ and 0.4 , respectively. The reason to make this assumption is that the dense materials (such as ice) have generally larger Poisson's ratios than its less dense counterpart (such as snow with $v=0$ ).

Fig. 11 shows the calculated Poisson's ratio of the amorphous PLA films listed in Table 2. It can be observed from this figure that the Poisson's ratio of all the amorphous polymers decreases by time. It also shows that the Poisson's ratio of the PDLLA film decreases more than other PLA films. This may be due to the higher porosity of the PDLLA film as shown in Fig. 4. In fact, degradation leads to cavities, making the material less dense. That means degradation leads to smaller Poisson's ratio for the amorphous polymers. 
The Poisson's ratio of the semi-crystalline polymers listed in Table 5-3 is shown in Fig. 12. It can be observed from this figure that the Poisson's ratio of the semi-crystalline polymers almost remains un-changed for an induction period then increases. The reason might be due to the increase in crystallinity of the polymer at the later stage of degradation which causes the polymer to become denser. Fitting tensile strength of degrading amorphous polymers using scaling law

The experimental data of the tensile strength of degrading polymers can be predicted by using the scaling relation of the porous metal foams from in the literature. This is particularly useful in the early stage of design when approximate analysis of components and structures is needed. The scaling relations for foam properties take the form (Ashby, 2000)

$$
\frac{P^{*}}{P_{s}}=\alpha\left\{\frac{\rho^{*}}{\rho_{s}}\right\}^{n}
$$

where $P$ is a property (subscripted s means property of the matrix material (solid metal) and superscript '*' denotes the property of the foam), $\alpha$ a constant and $n$ a fixed exponent - they depend on the structure of the foam and $\rho$ is the density. For instance, the tensile strength of the porous metal foam scale with its density $\rho\left(\mathrm{Mg} / \mathrm{m}^{3}\right)$ as

$$
\sigma_{t} \approx \alpha \sigma_{c, s}\left(\frac{\rho}{\rho_{s}}\right)^{3 . / 2}
$$

where $\alpha$ has a value between 0.1 and 1.4, $\sigma_{c, s}$ is the initial compressive strength of the matrix (solid metal), $\rho / \rho_{s}$ is the material relative density and $\rho_{s}$ is density of the matrix.

The relative density of a degrading amorphous polymer due to the presence of cavities can be expressed in terms of porosity as

$$
\frac{\rho}{\rho_{0}}=1-f_{I}^{(1)}
$$

where $f_{I}^{(1)}$ is the density of cavities (porosity) that can be obtained from Eq. (16), $\rho$ is the density of degrading polymer and $\rho_{0}$ is the polymer density before degradation.

Using above relations the tensile strength of a degrading amorphous polymer can be predicted by 


$$
\sigma_{t}=\alpha \sigma_{t 0}\left(1-f_{I}^{(1)}\right)^{n}
$$

where $\sigma_{t 0}$ is the initial tensile strength, $\alpha$ has a value of 1 and $n$ takes different values depends on the polymer structure.

Applying Eq. (24) on amorphous PLLA, PDLA, PDLLA and PDLA/PLLA films listed in Table 2 predicts their tensile strength as Fig.13. The PLA films have the same porosities $\rho_{C}$ as what obtained in section 4 (Fig. 4). That means the same radius of cavity $r$ as what used in section 4 for predicting the Young modulus of the PLA films is used here for the prediction of the tensile strength of the PLA films. The values of $r$ and $n$ have been given in Table 4 .

It can be observed from Fig. 23 that the scaling law can capture the trend of the experimental tensile strength of the amorphous PLA films fairly well. Table 4 shows that PLA films with a higher porosity have a higher value of $n$. In fact, the value of $n$ governs by the density of the degrading polymer which depends on the porosity of the polymer.

\subsection{Sensitivity analysis}

The sensitivity analysis shows that the tensile strength of the PLA films decreases faster during the degradation time when $r$ remains constant but $n$ increases by $30 \%$ as shown in Fig. 24. This indicates that the tensile strength is not very sensitive to $n$.

However, when $n$ remains constant but $r$ increases by $30 \%$, then the tensile strength of the PLA films decreases much faster than when $r$ remains constant but $r$ increases by $30 \%$ as shown in Fig.15. This indicates that the tensile strength is sensitive to $r$ rather than $n$.

\section{Conclusions}

A complete constitutive law consisting of Model One and Model Two for elastic properties and the scaling law for strength is presented to predict the change in elastic moduli, Poisson's ratio and ultimate tensile strength of bioresorbable polymers due to biodegradation. The elastic moduli and Poisson's ratio of semi-crystalline polymers having both initial and scission-induced crystallinity were predicted by Model One which was also reduced to predict the elastic moduli and Poisson's ratio of the amorphous polymers referred to as Model Two. The scaling law (that was derived based on the scaling relations for metal foam 
properties) was used for fitting the tensile strength experimental data of degrading amorphous bioresorbable polymers.

The results of the study showed that the constitutive law could successfully capture the trend of the changes in observed mechanical properties of degrading bioresorbable polymers. It also showed that the radius of cavities, $r$, which was the only fitting parameter in predicting the elastic moduli of degrading polymers and used to calculate the porosity of such polymers, had a value between $0.5-1.5 \mathrm{~nm}$ for amorphous polymers and 1.5-4.5 for semi-crystalline polymers. Meanwhile, it was found out that the scaling law was very sensitive to $r$ but not much to another fitting parameter, $n$, that as a fixed exponent could take different values depending on the polymer structure and was increasing when porosity of the degrading polymers was decreasing.

Furthermore, the results showed that the degrading amorphous polymers with higher porosity had a lower Poisson's ratio. For instance, the Poisson's ratio of the four amorphous PLA films almost with $10 \%, 30 \%, 70 \%$ and $100 \%$ porosities after 2 year hydrolytic degradation were decreased to about $4 \%, 13 \%, 23 \%$ and $30 \%$ respectively. The results also showed that the Poisson's ratio of the semi-crystalline polymers almost remained un-changed for an induction period then increased. The reason might be due to the increase in the crystallinity of the polymeric films which causes the polymers to become denser. In this study, the Poisson's ratio of the matrix and crystal phases was assumed to be 0.35 and 0.4 , respectively. The reason to make this assumption was that the dense materials (such as ice) have generally larger Poisson's ratios than its less dense counterpart (such as snow, $v=0$ ).

Acknowledgement: This work is supported by a PhD studentship for Samami by the College of Science of Engineering, the University of Leicester, which is gratefully acknowledged.

\section{Appendix-Nomenclature}

$\Omega^{(\alpha)}$ Inhomogeneities $(\alpha=1,2)$

$C_{I}^{(\alpha)} \quad$ Elastic stiffness of inhomogeneities

$K_{I}^{(\alpha)} \quad$ Bulk modulus of inhomogeneities

$\mu_{I}^{(\alpha)} \quad$ Shear modulus of inhomogeneities

$f_{I}^{(\alpha)} \quad$ Volume fractions of inhomogeneities

$\bar{\sigma}_{I}^{(\alpha)} \quad$ Average stress of inhomogeneities 
$\bar{\varepsilon}_{I}^{(\alpha)} \quad$ Average strain of inhomogeneities

$f_{M} \quad$ Volume fraction of matrix

$C_{M} \quad$ Elastic stiffness of matrix

$K_{M} \quad$ Bulk modulus of matrix

$\mu_{M} \quad$ Shear modulus of matrix

$\bar{\sigma}_{M} \quad$ Average stress of matrix

$\bar{\varepsilon}_{M} \quad$ Average strain of matrix

$C \quad$ Effective elastic stiffness of material

$K \quad$ Bulk modulus of the material

$\mu \quad$ Shear moduli of material

$v \quad$ Poisson's ratio of material

$\bar{\sigma} \quad$ Average stress of material

$\sigma^{0} \quad$ Uniform applied stress

$\varepsilon_{k k}^{0} \quad$ Remote hydrostatic pressure

$\varepsilon_{12}^{0} \quad$ Remote shear strain

$r \quad$ Radius of cavities

$\bar{M}_{n} \quad$ Number averaged molecular weight normalised by its initial value

$M_{n 0} \quad$ Initial number average molecular weight $(\mathrm{g} / \mathrm{mol})$

$M_{\text {unit }}$ Molecular weight of the repeating unit of the polymer chain $(\mathrm{Kg} / \mathrm{mol})$

$C_{e 0} \quad$ Initial concentration of ester bonds $(\mathrm{mol} / \mathrm{m} 3)$

$\alpha, \beta \quad$ Empirical parameters for oligomer production (dimensionless)

$\rho \quad$ Density $(\mathrm{g} / \mathrm{m} 3)$ of degrading polymer

$\rho_{0} \quad$ Initial Polymer density (g/m3) (before degradation)

$m$ Degree of polymerization of the oligomers (dimensionless)

$R_{S} \quad$ Total number of chain-scissions per unit volume ( $\left.\mathrm{mol} / \mathrm{m} 3\right)$

$\bar{R}_{S} \quad$ Total number of chain-scission normalised by initial number of ester bond per unit volume

$X_{c 0} \quad$ Initial degree of crystallinity

$X_{c} \quad$ Scission-induced crystallinity

$\omega \quad$ Number of ester units of crystalline phase per unit volume

$N_{\text {chain } 0}$ Initial number of molecular chains per unit volume including crystalline phase

$E_{0} \quad$ Initial Young modulus

$E_{I}^{(2)} \quad$ Young modulus of the crystals to fit the data

$P^{*} \quad$ A property of the metal foam

$P_{s} \quad$ A property of the matrix of metal foam 
$\rho_{s} \quad$ Density of the matrix of metal foam

$f_{I}^{(1)} \quad$ Density of cavities (porosity)

$\alpha \quad$ A constant

$\sigma_{t} \quad$ Tensile strength of the porous metal foam

$\sigma_{t 0} \quad$ Initial tensile strength

$\sigma_{c, s} \quad$ Initial compressive strength of the matrix of metal foam

\section{References}

[1] ABOUDI, J. 1991. Mechanics of composite materials: a unified micromechanical approach, Amsterdam, Elsevier.

[2] ASHBY, M. F. 2000. Metal foams: a design guide, Boston, Mass, ButterworthHeinemann

[3] ASHBY, M. F. \& JONES, D. R. H. 2005. Engineering materials 1: an introduction to properties, applications and design, Amsterdam, Elsevier Butterworth-Heinemann.

[4] BOUAPAO, L., TSUJI, H., TASHIRO, K., ZHANG, J. \& HANESAKA, M. 2009. Crystallization, spherulite growth, and structure of blends of crystalline and amorphous poly(lactide)s. Polymer, 50, 4007-4017.

[5] BUCHANAN, F., INSTITUTE OF MATERIALS, M. \& MINING 2008. Degradation rate of bioresorbable materials: prediction and evaluation, Cambridge, Woodhead Publishing on behalf of the Institute of Materials, Minerals and Mining.

[6] BUDIANSKY, B. 1965. On the elastic moduli of some heterogeneous materials. Journal of the Mechanics and Physics of Solids, 13, 223-227.

[7] DUEK, E. A. R., ZAVAGLIA, C. A. C. \& BELANGERO, W. D. 1999. In vitro study of poly(lactic acid) pin degradation. Polymer, 40, 6465-6473.

[8] ESHELBY, J. D. 1957. The Determination of the Elastic Field of an Ellipsoidal Inclusion, and Related Problems. Proceedings of the Royal Society of London. Series A. Mathematical and Physical Sciences, 241, 376-396. 
[9] GLEADALL, A., PAN, J. \& ATKINSON, H. 2012. A simplified theory of crystallisation induced by polymer chain scissions for biodegradable polyesters. Polymer Degradation and Stability, 97, 1616-1620.

[10] GLEADALL, A. 2014.Modelling degradation of biodegradable polymers and their mechanical properties. $\mathrm{PhD}$ thesis. University of Leicester

[11] HAN, X. \& PAN, J. 2008. A Model for Simultaneous Crystallisation and Biodegradation of Biodegradable Polymers.

[12] HASHIN, Z. 1962. The Elastic Moduli of Heterogeneous Materials. Journal of Applied Mechanics, 29, 143-150.

[13] HASHIN, Z. \& SHTRIKMAN, S. 1962. A variational approach to the theory of the elastic behaviour of polycrystals. Journal of the Mechanics and Physics of Solids, 10, $343-352$.

[14] HILL, R. 1963. Elastic properties of reinforced solids: Some theoretical principles. Journal of the Mechanics and Physics of Solids, 11, 357-372.

[15] KACHANOV, M., TSUKROV, I. \& SHAFIRO, B. 1994. Effective Moduli of Solids With Cavities of Various Shapes. Applied Mechanics Reviews, 47, S151-S174.

[16] LAM, K. H., NIEUWENHUIS, P., MOLENAAR, I., ESSELBRUGGE, H., FEIJEN, J., DIJKSTRA, P. J. \& SCHAKENRAAD, J. M. 1994. Biodegradation of porous versus non-porous poly(L-lactic acid) films. Journal of Materials Science: Materials in Medicine, 5, 181-189.

[17] MACKENZIE, J. K. 1950. The Elastic Constants of a Solid containing Spherical Holes. Proceedings of the Physical Society. Section B, 63, 2-11.

[18] MORI, T. \& TANAKA, K. 1973. Average stress in matrix and average elastic energy of materials with misfitting inclusions. Acta Metallurgica, 21, 571-574.

[19] MURA, T. 1987. Micromechanics of defects in solids, Lancaster, Nijhoff.

[20] PAN, J. 2014. Modelling Degradation of Bioresorbable Polymeric Medical Devices, Burlington, Woodhead Publishing. 
[21] SAHA, S. K. \& TSUJI, H. 2006. Effects of rapid crystallization on hydrolytic degradation and mechanical properties of poly( 1-lactide-co- $\varepsilon$-caprolactone). Reactive and Functional Polymers, 66, 1362-1372.

[22] SHODJA, H. M., RAD, I. Z. \& SOHEILIFARD, R. 2003. Interacting cracks and ellipsoidal inhomogeneities by the equivalent inclusion method. Journal of the Mechanics and Physics of Solids, 51, 945-960.

[23] TSUJI, H. 2000. In vitro hydrolysis of blends from enantiomeric poly(lactide)s Part 1. Well-stereo-complexed blend and non-blended films. Polymer, 41, 3621-3630.

[24] TSUJI, H. 2002. Autocatalytic hydrolysis of amorphous-made polylactides: effects of 1lactide content, tacticity, and enantiomeric polymer blending. Polymer, 43, 17891796.

[25] TSUJI, H. 2003. In vitro hydrolysis of blends from enantiomeric poly(lactide)s. Part 4: well-homo-crystallized blend and nonblended films. Biomaterials, 24, 537-547.

[26] TSUJI, H. \& DEL CARPIO, C. A. 2003. In vitro hydrolysis of blends from enantiomeric poly(lactide)s. 3. Homocrystallized and amorphous blend films. Biomacromolecules, 4, 7-11.

[27] TSUJI, H. \& IKADA, Y. 2000. Properties and morphology of poly( 1-lactide) 4. Effects of structural parameters on long-term hydrolysis of poly( 1-lactide) in phosphatebuffered solution. Polymer Degradation and Stability, 67, 179-189.

[28] TSUJI, H., IKARASHI, K. \& FUKUDA, N. 2004. Poly( 1-lactide): XII. Formation, growth, and morphology of crystalline residues as extended-chain crystallites through hydrolysis of poly( 1-lactide) films in phosphate-buffered solution. Polymer Degradation and Stability, 84, 515-523.

[29] TSUJ, H., MIZUNO, A. \& IKADA, Y. 2000. Properties and morphology of poly(Llactide). III. Effects of initial crystallinity on long-term in vitro hydrolysis of high molecular weight poly(L-lactide) film in phosphate-buffered solution. Journal of Applied Polymer Science, 77, 1452-1464. 
[30] TSUJ, H. \& MURAMATSU, H. 2001. Blends of aliphatic polyesters: V non-enzymatic and enzymatic hydrolysis of blends from hydrophobic poly( 1-lactide) and hydrophilic poly(vinyl alcohol). Polymer Degradation and Stability, 71, 403-413.

[31] WALPOLE, L. J. 1966. On bounds for the overall elastic moduli of inhomogeneous systems-I. Journal of the Mechanics and Physics of Solids, 14, 151-162.

[32] WEIR, N. A., BUCHANAN, F. J., ORR, J. F. \& DICKSON, G. R. 2004. Degradation of poly-L-lactide. Part 1: in vitro and in vivo physiological temperature degradation. Proceedings of the Institution of Mechanical Engineers.Part H, Journal of engineering in medicine, 218, 307-319.
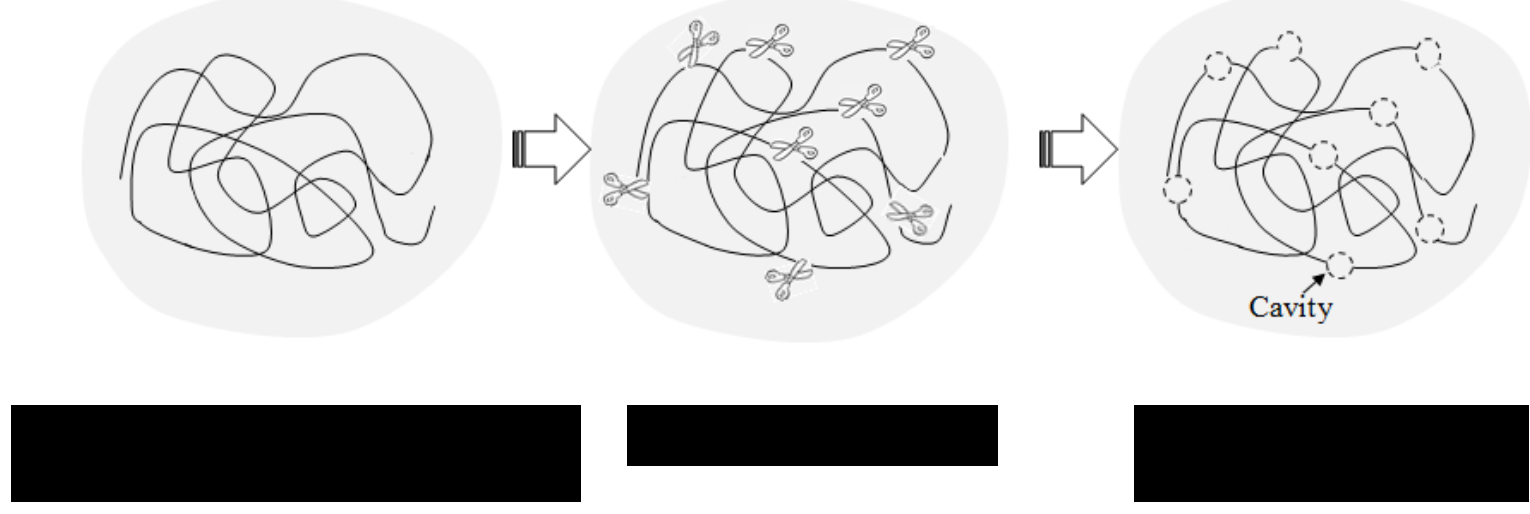

Fig. 1: Assumption of using cavities to represent polymer chain cleavages 

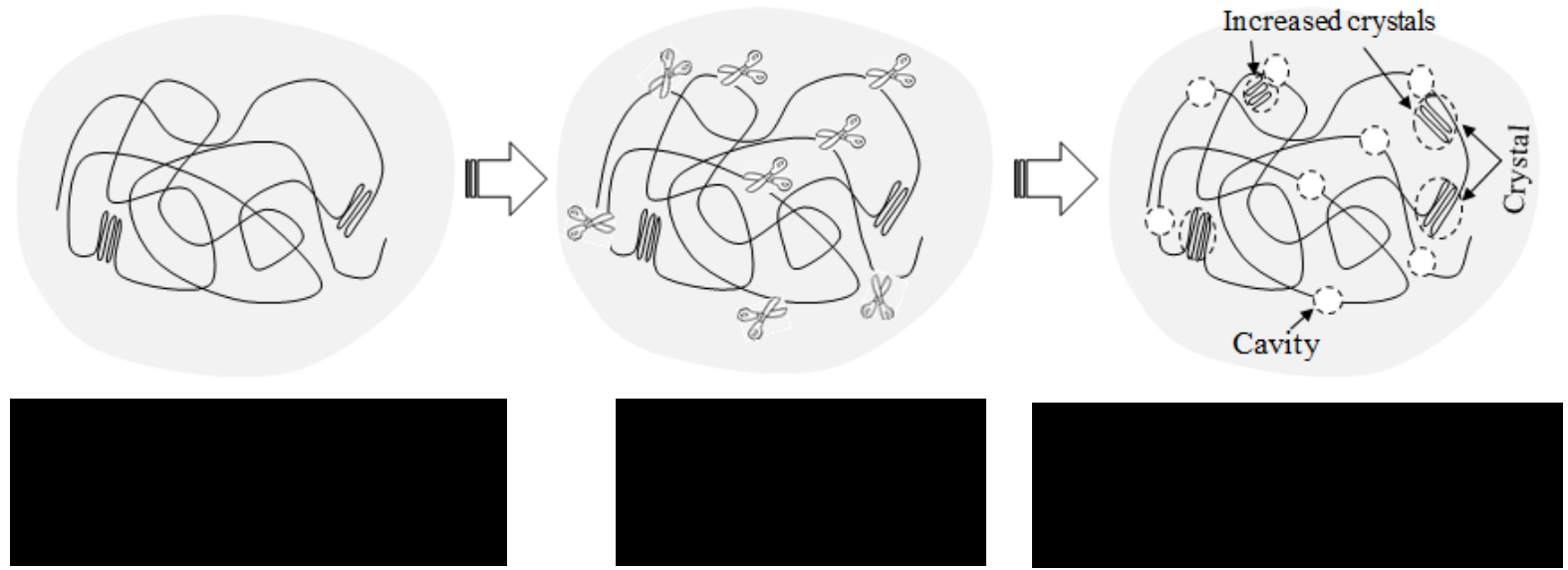

Fig.2: Assumption of using cavities and crystals to represent a degrading semicrystalline polymer

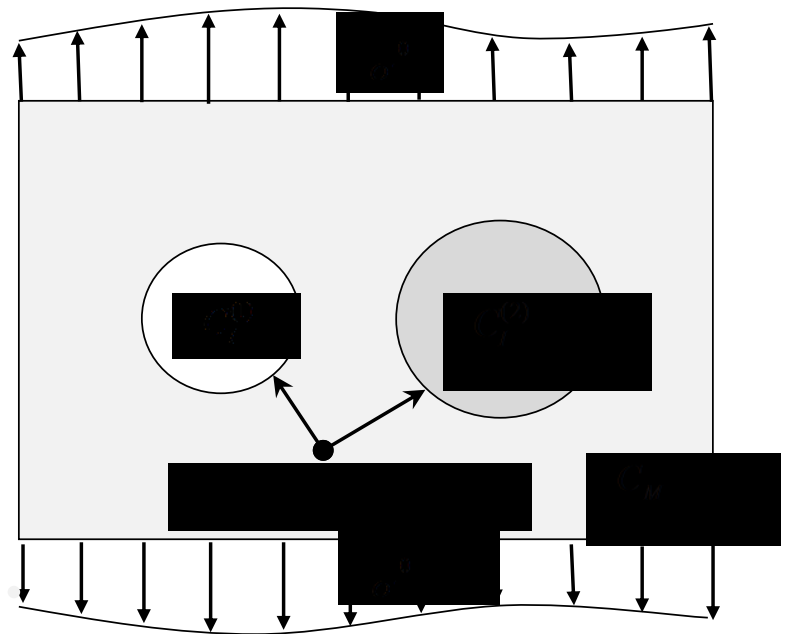

Fig. 3: The RVE containing two inhomogeneities with different stiffness (adapted from (Shodja et al., 2003) 


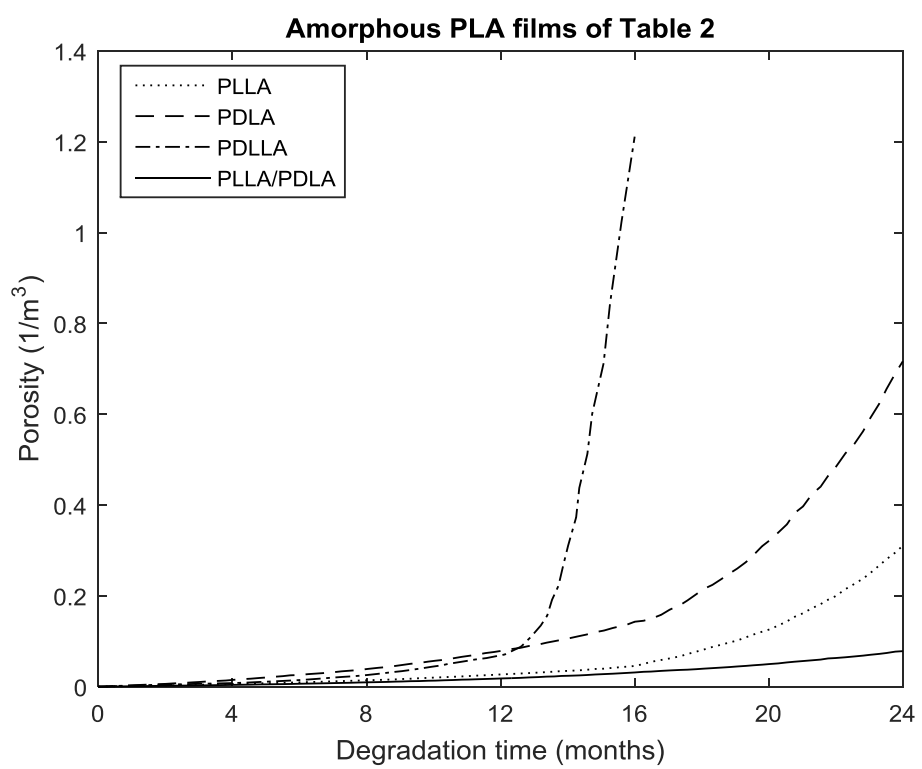

Fig.4: Porosity of PLA films of Table 2 as a function of time tested by Tsuji et al (Tsuji, 2002)

(a) PLLA

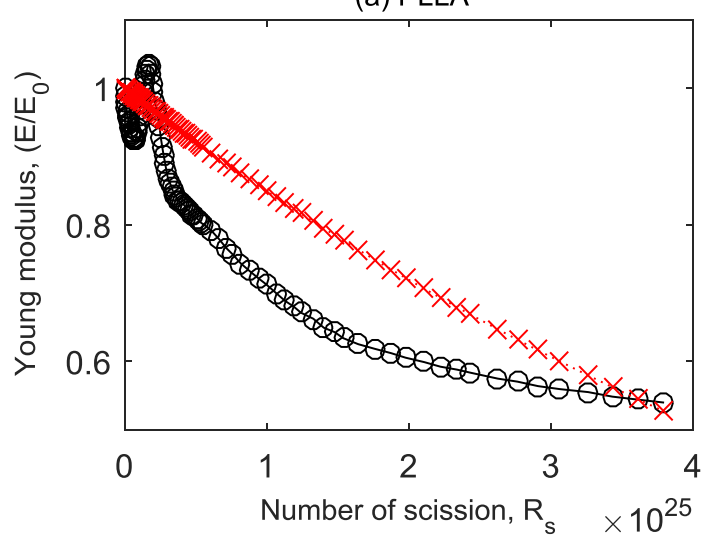

(c) PDLLA

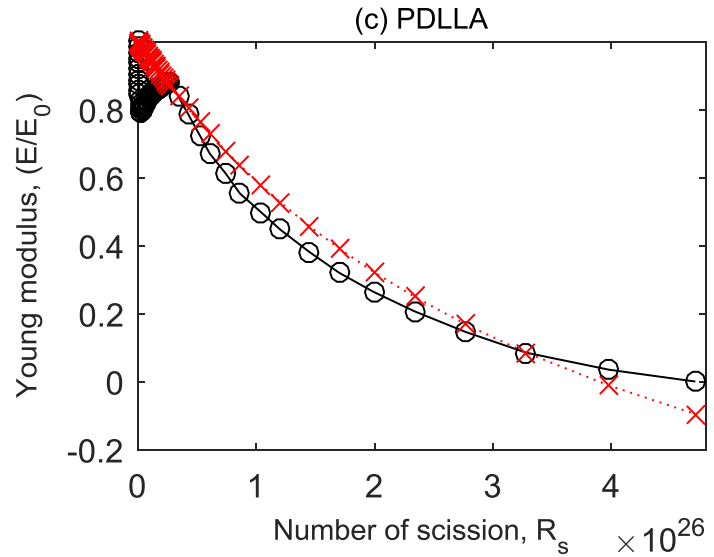

(b) PDLA

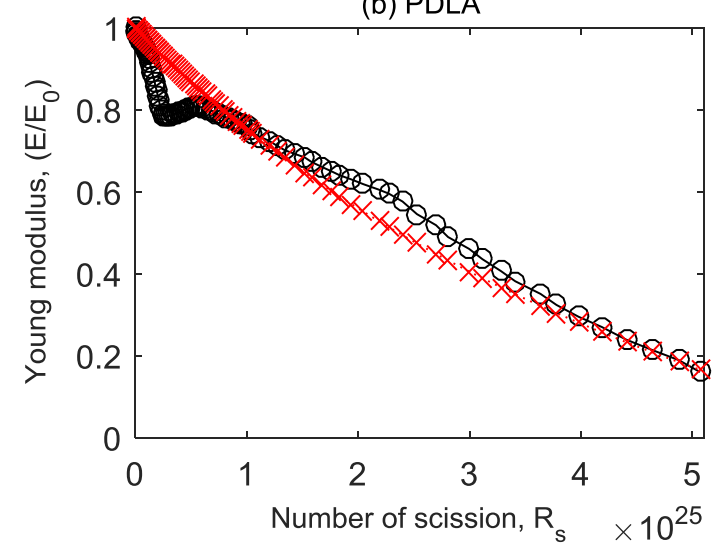

(d) PDLA/PLLA

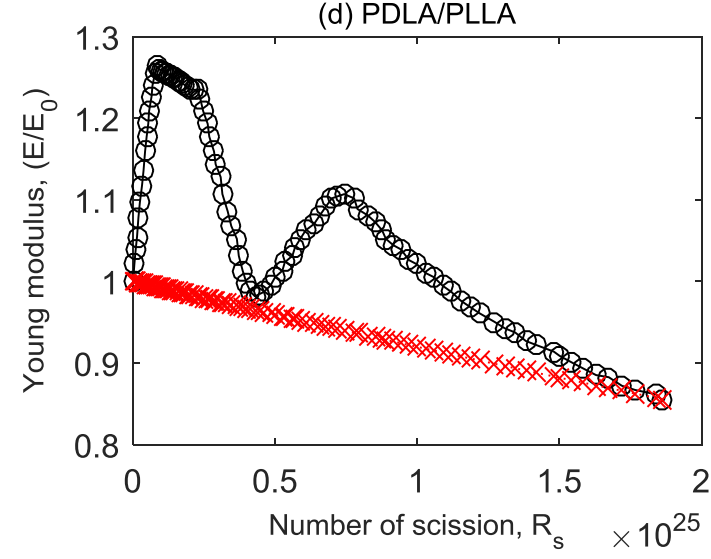

- Experiment $\cdots \cdots \cdots \cdots \cdot$ Model Two (with MTS)

Fig.5: Fitting of Young modulus as a function of degradation time using MTS model with experimental data obtained by Tsuji et al (Tsuji, 2002) for amorphous PLLA, PDLA, PDLLA and PLLA/PDLA films 

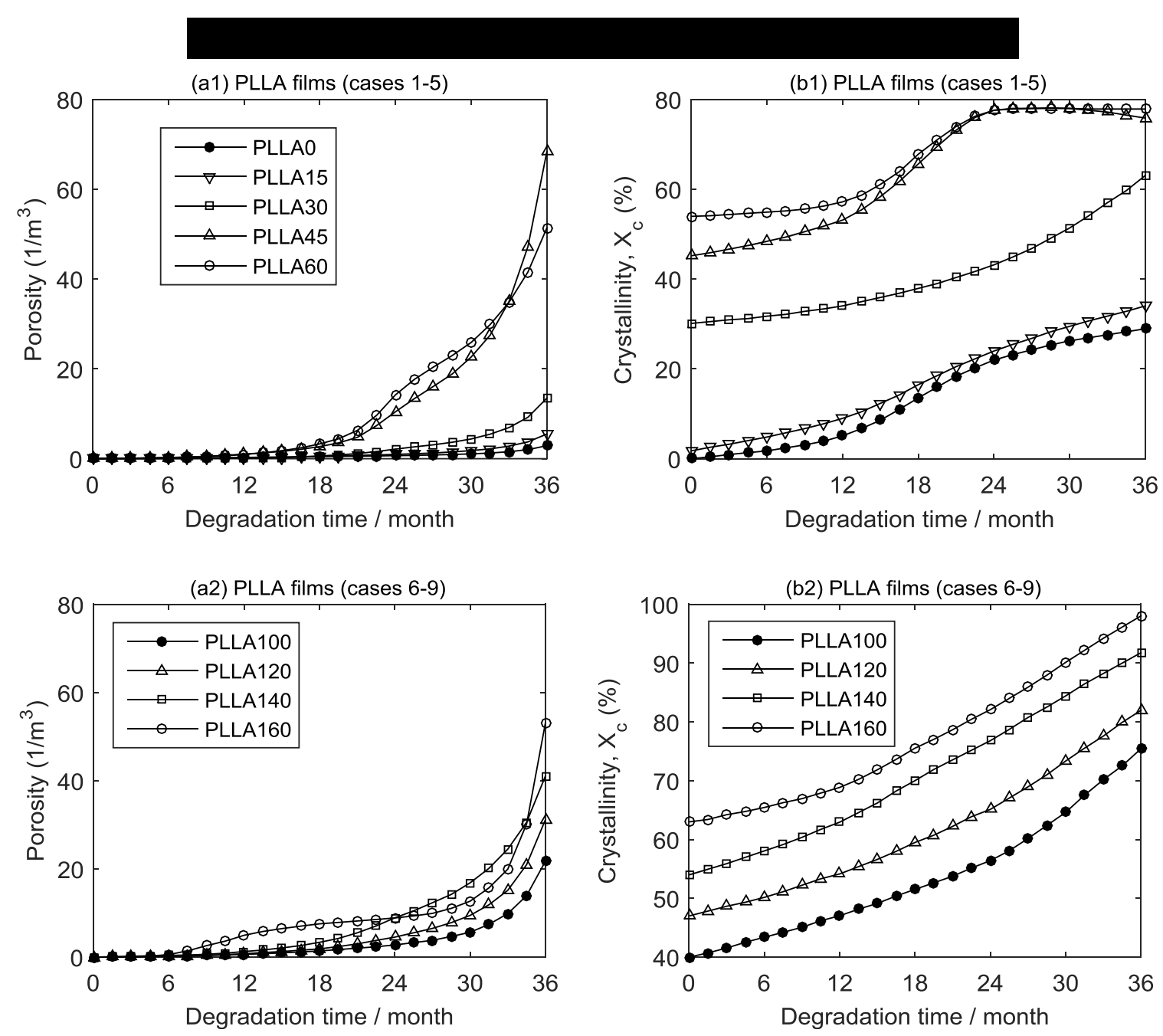

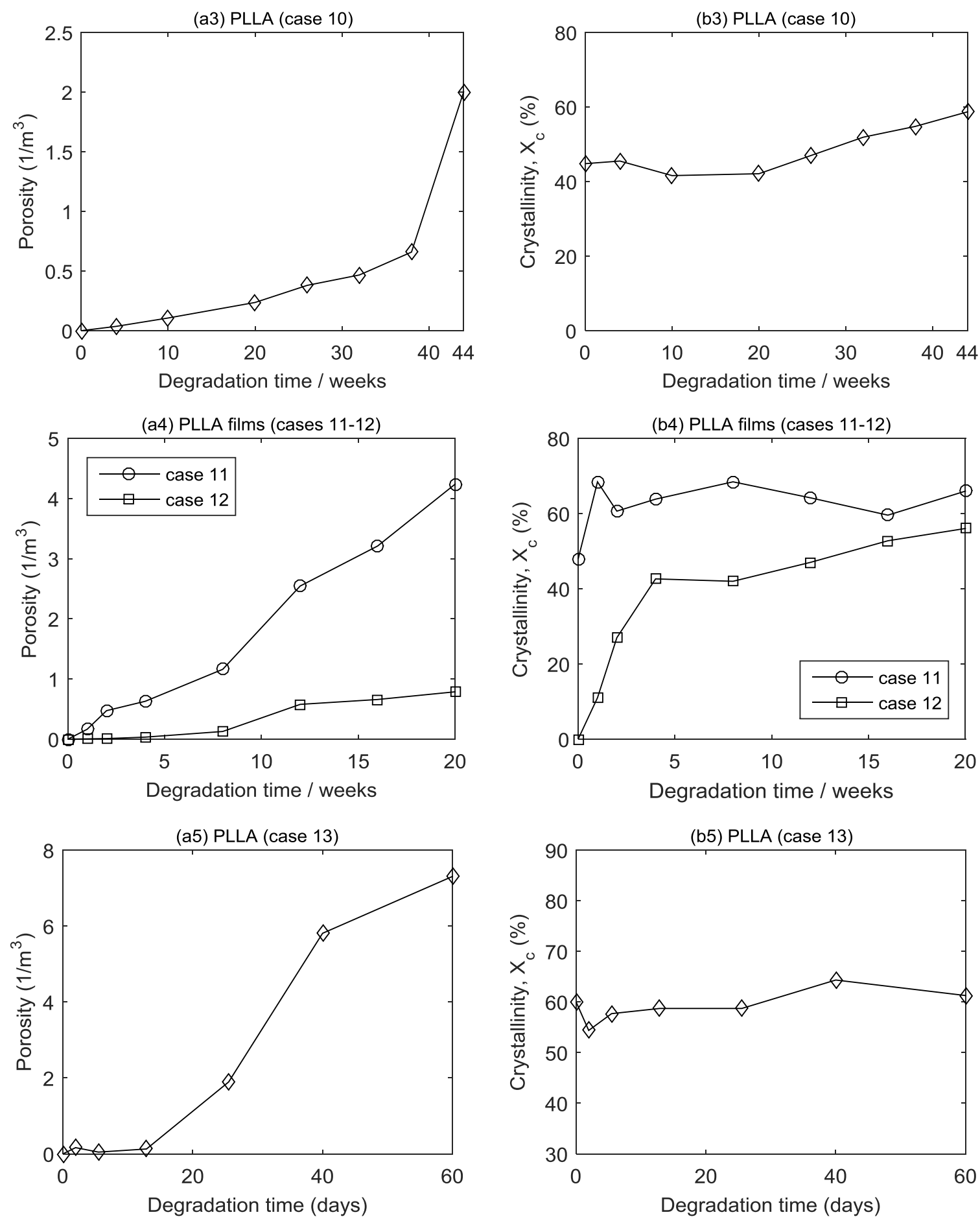

Fig. 6: Crystallinity (experimental data) and porosity (calculated) of semicrystalline PLLA films (cases 1-13 of Table 3) 

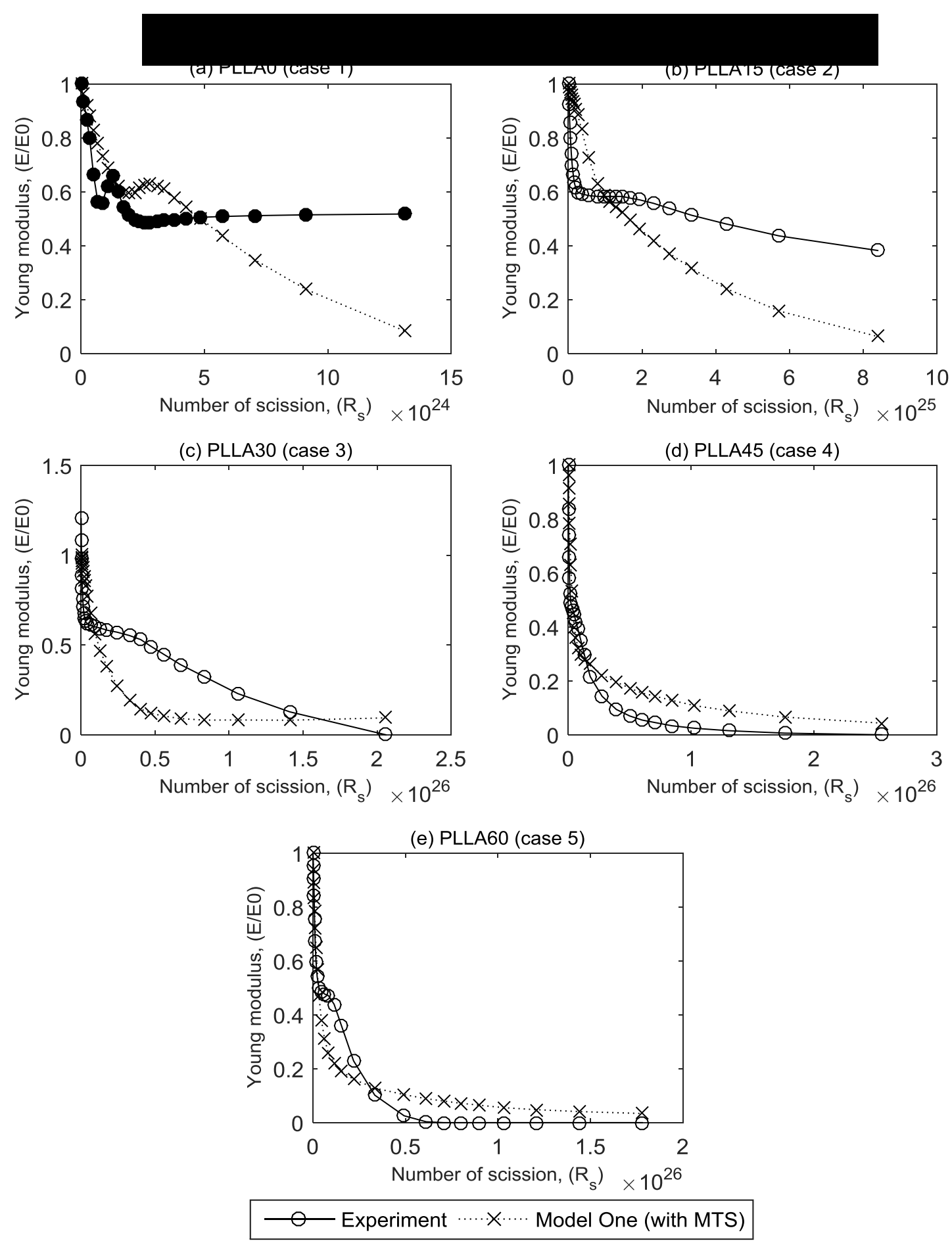

Fig. 7: Fitting of experimental data of Young modulus of polymer cases 1-5 of Table 3 using Model One with MTS concentration factors. The experimental data were taken from (Tsuji et al., 2000) 

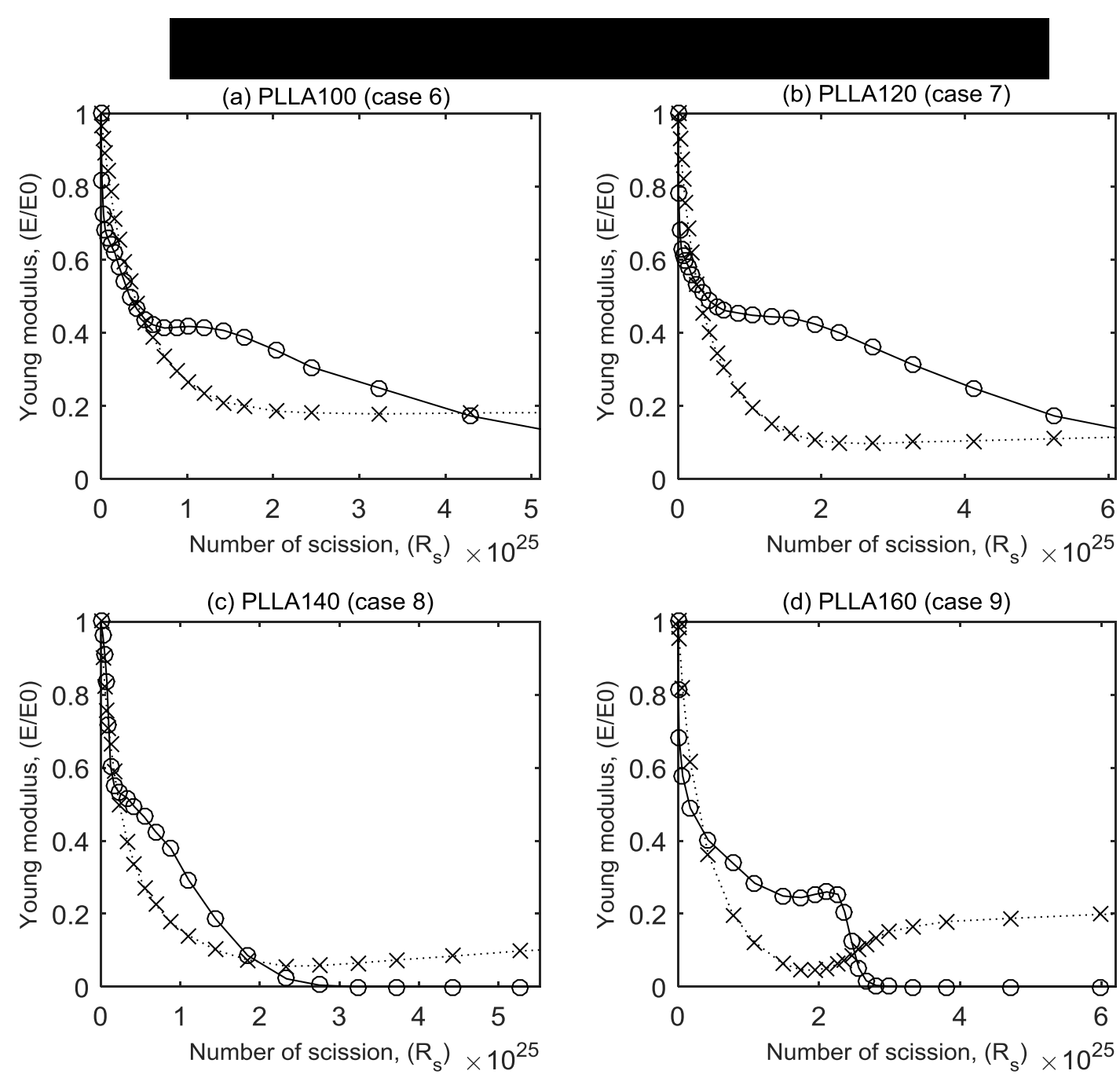

- Experiment $\cdots \cdots \times \cdots \cdot$ Model One (with MTS)

Fig. 8: Fitting of experimental data of Young modulus of semi-crystalline PLLA polymers of cases 6-9 of Table 3 using MTS concentration factors in Model One. The experimental data were taken from (Tsuji and Ikada, 2000) 

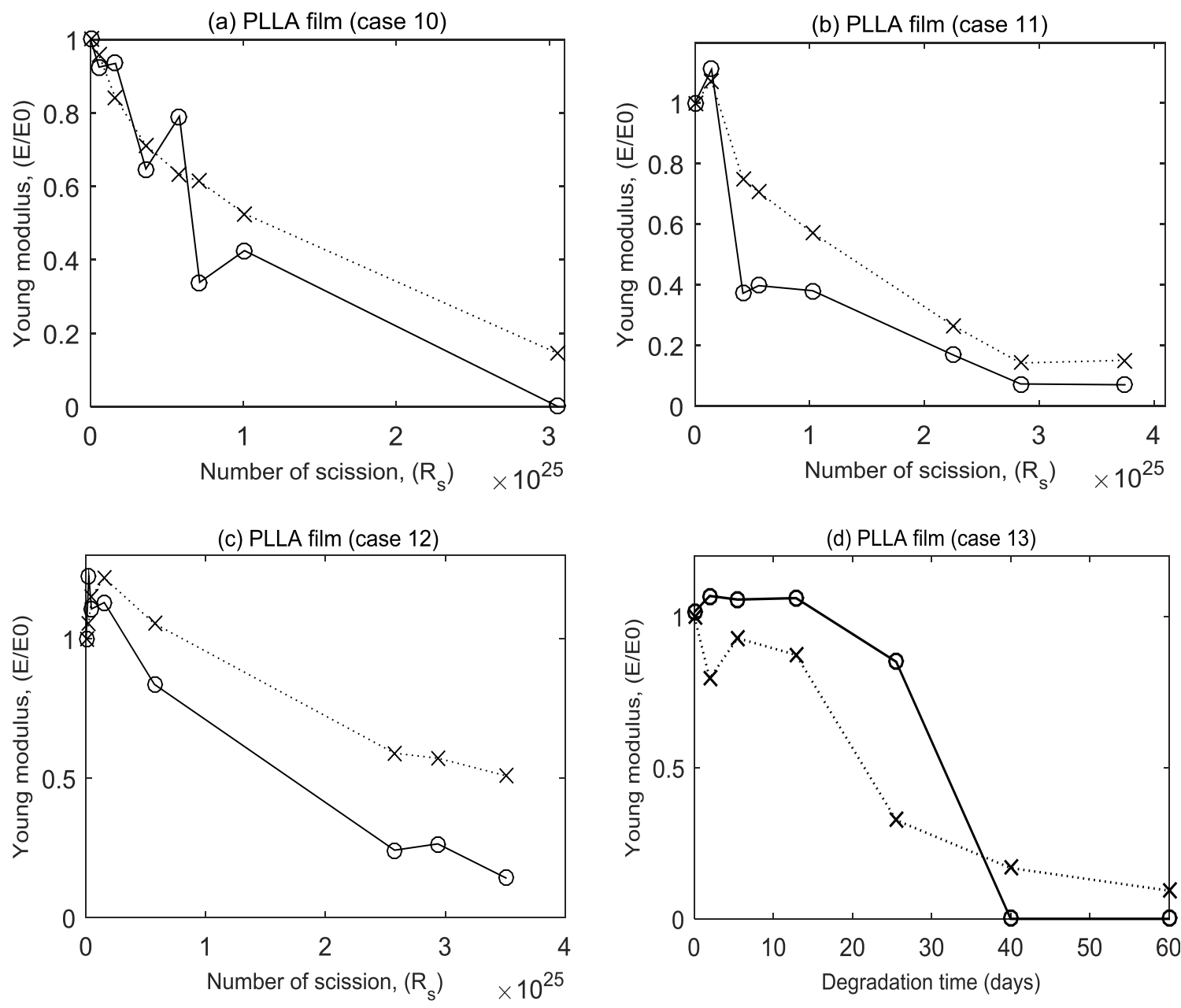

- Experiment $\cdots \cdots \times \cdots \cdot$ Model One (with MTS)

Fig. 9: Fitting of experimental data of Young modulus of semi-crystalline PLLA polymer (case 10-13 of Table 3) using Model One with MTS concentration factors 
Young's modulus of semi-crystalline PLLA films (cases 6-9 of Table 3)
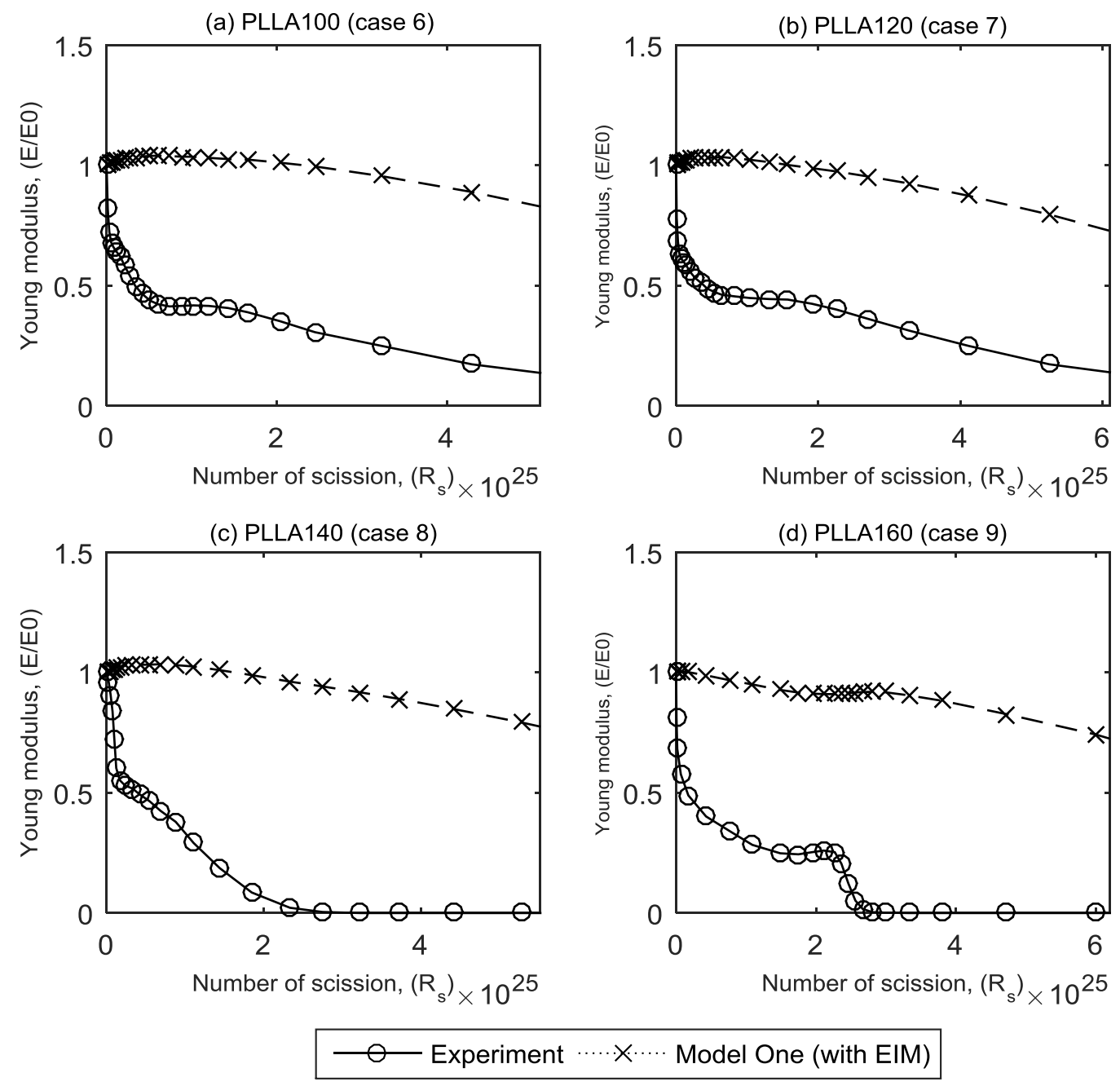

Fig. 10: Fitting of experimental data of Young modulus of semi-crystalline PLLA polymers (cases 6-9 of Table 3) using Model One with EIM concentration factors 


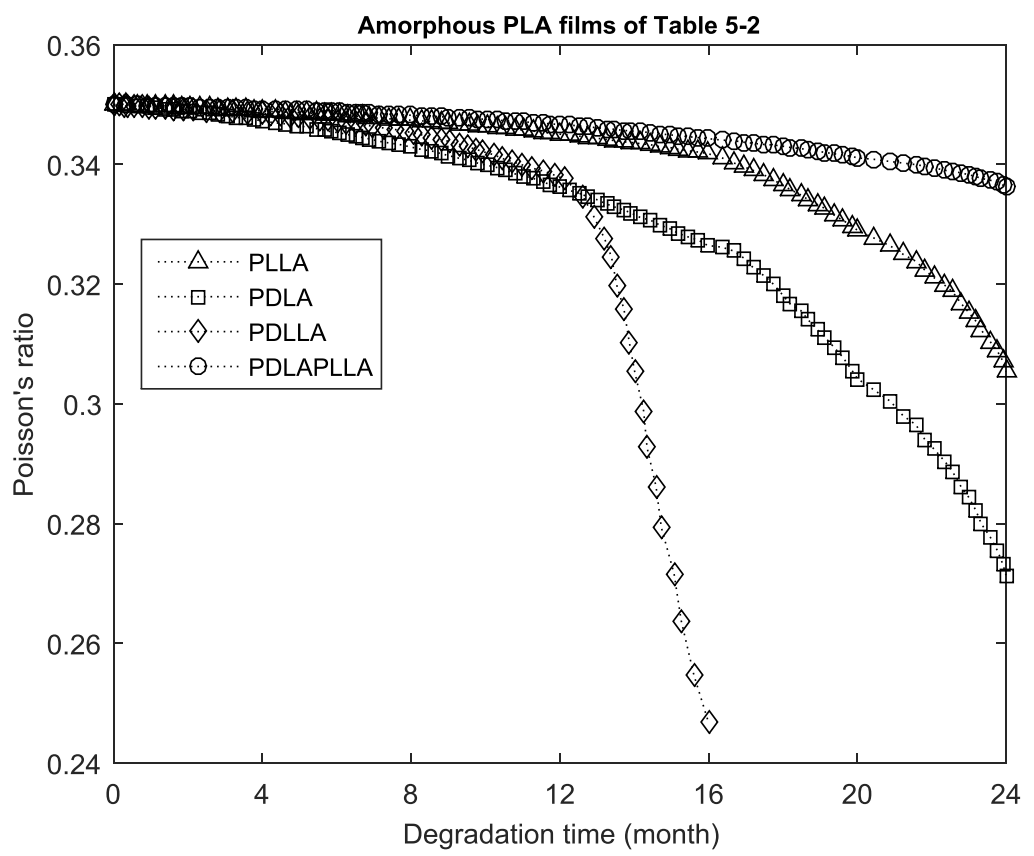

Fig. 11: Calculated Poisson's ratio of amorphous PLLA, PDLA, PDLLA and PDLA/PLLA films (listed in table 2) as function of degradation time over 24 months 

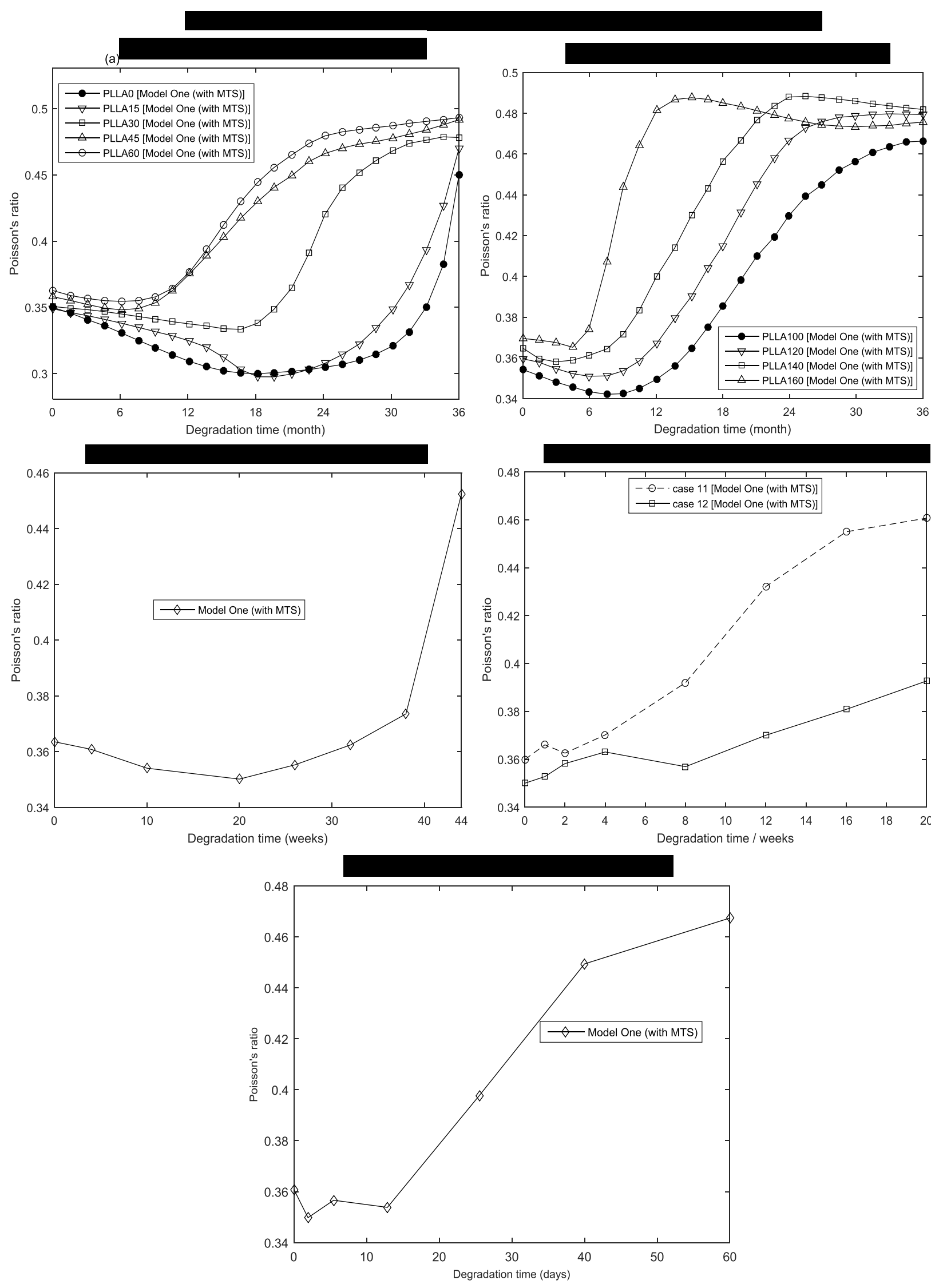

Fig. 12: Calculated Poisson's ratio of semi-crystalline PLLA films of cases 1-13 of

Table 3 


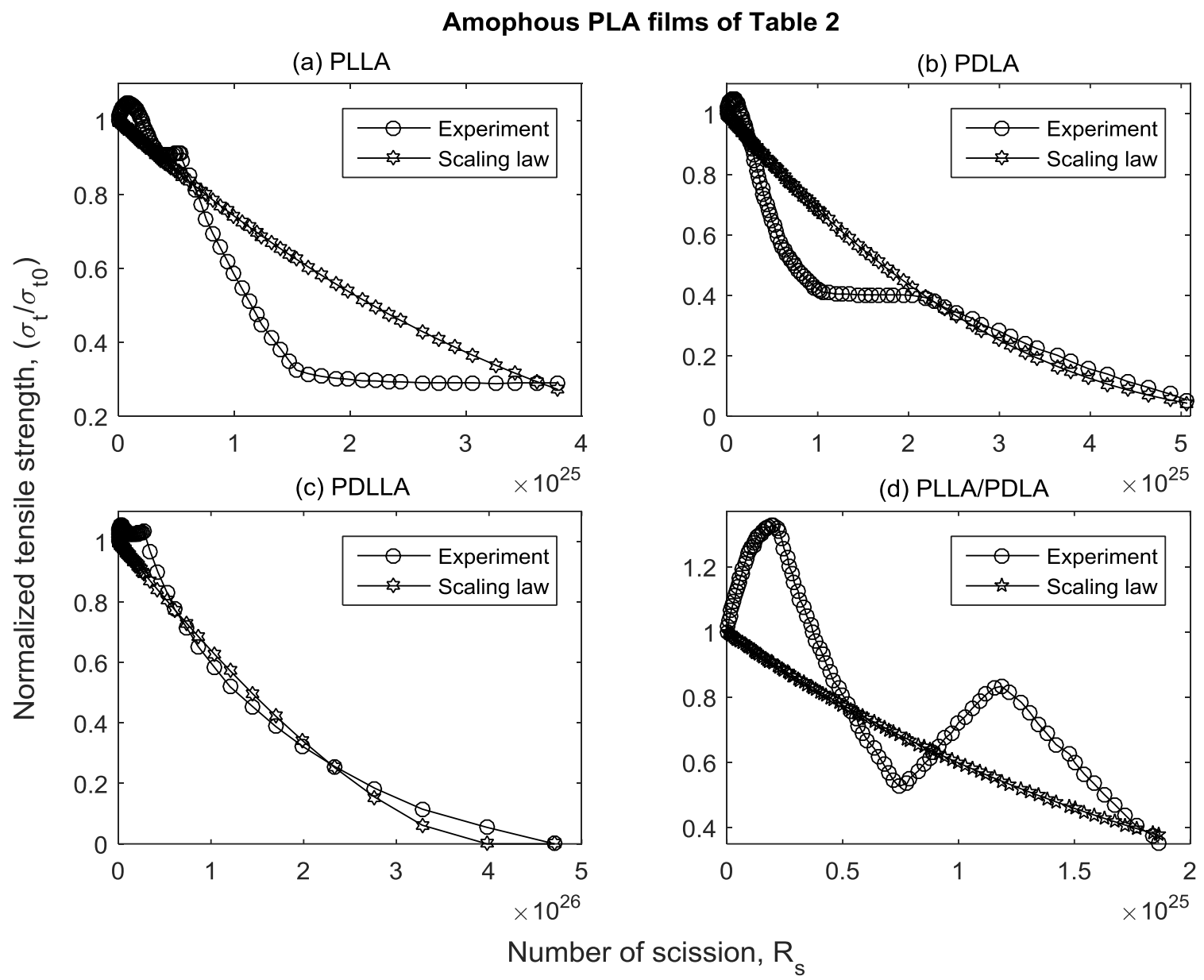

Fig.13: Fitting of the tensile strength of the amorphous PLA films of Table 2 using scaling law 
Amophous PLA films of Table 2

(a) PLLA

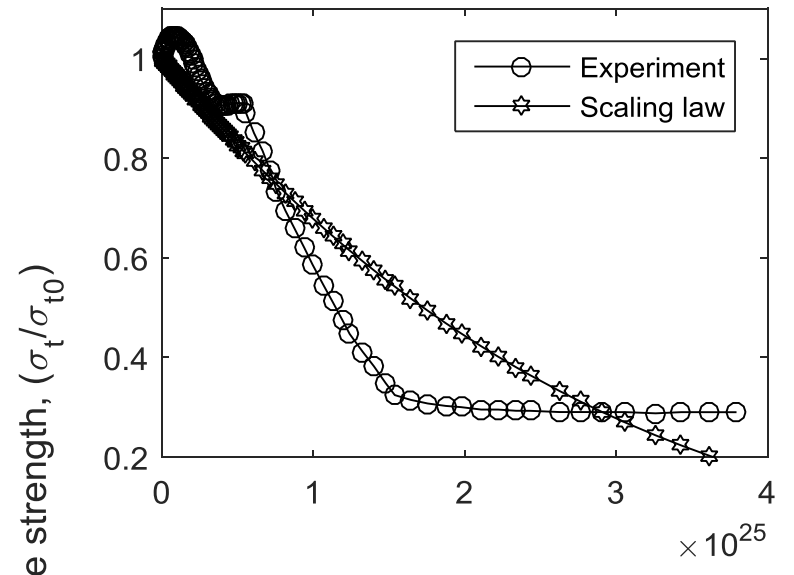

(c) PDLLA

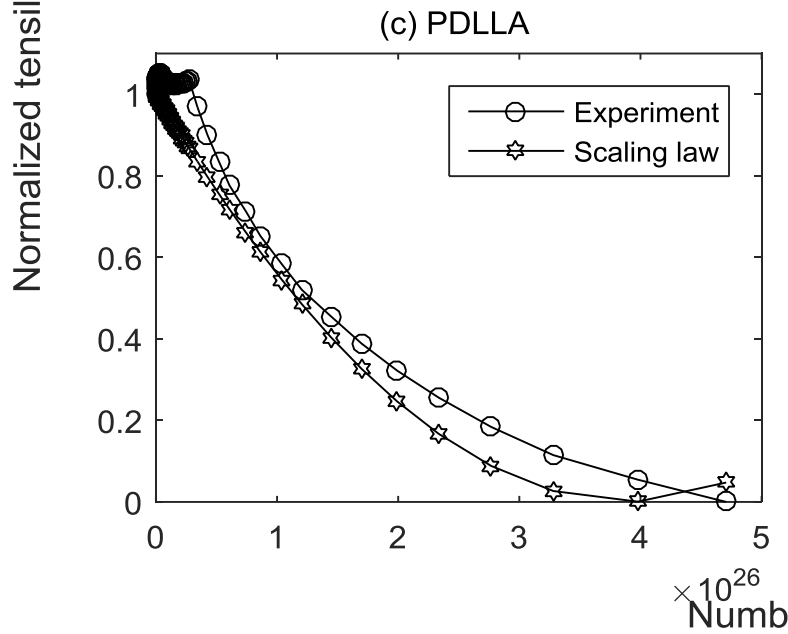

Fig. 14: Tensile strength of the amorphous PLA films of Table2 when $r$ remains constant but $n$ increases by $30 \%$ (b) PDLA
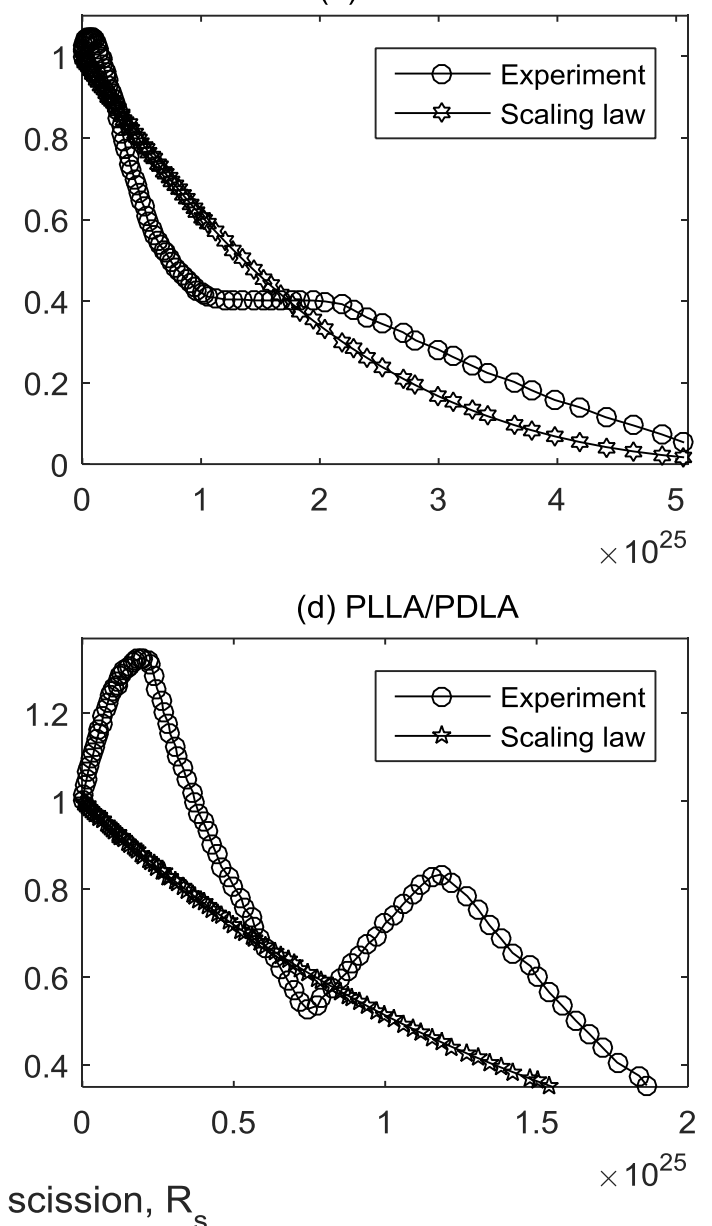

(d) PLLA/PDLA 


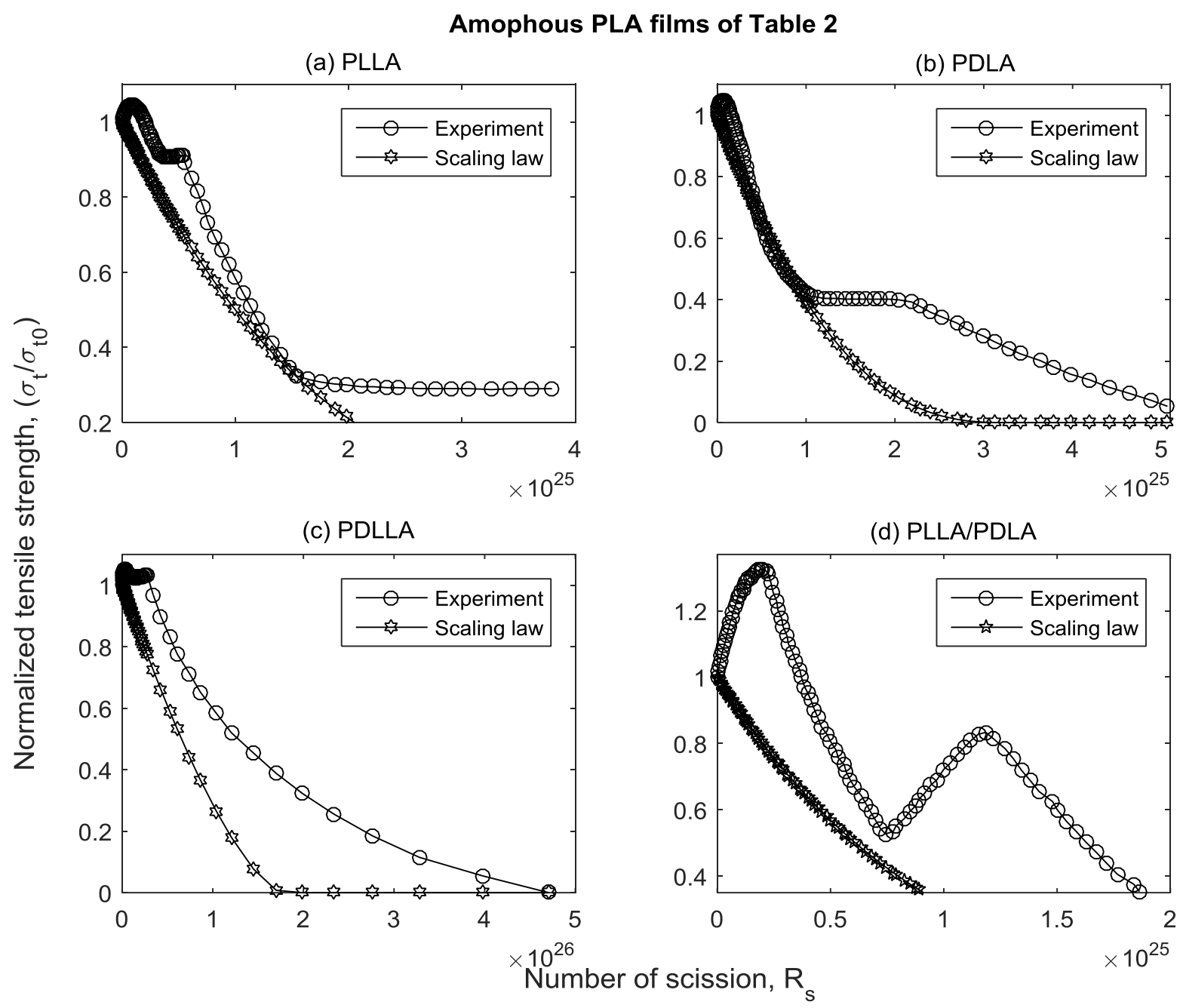

Fig.15: Tensile strength of the amorphous PLA films of Table2 when $r$ increases by $30 \%$ but $n$ remains constant 
Table 1: Concentration factor of EIM and MTS schemes for elastic spherical inclusions (Aboudi, 1991, Mura, 1987)

\begin{tabular}{c|cc}
\hline $\begin{array}{c}\text { Concentration } \\
\text { factors }\end{array}$ & EIM & MTS \\
\hline $\bar{\varepsilon}_{\mathrm{I}_{12}}^{(1)} / \varepsilon_{12}^{0}$ & $\frac{15\left(1-v_{0}\right)}{\left(7-5 v_{0}\right)}$ & $\frac{1}{\left[\frac{-6\left(K_{M}+2 \mu_{M}\right)\left(1-f_{I}^{(1)}\right)}{5\left(3 K_{M}+4 \mu_{M}\right)}+1\right]}$ \\
$\bar{\varepsilon}_{\mathrm{I}_{\mathrm{kk}}^{(1)} / \varepsilon_{\mathrm{kk}}^{0}}$ & $\frac{3\left(v_{0}-1\right)}{2\left(2 v_{0}-1\right)}$ & $\frac{1}{\left.\frac{-3\left(1-f_{I}^{(1)}\right) K_{M}}{\left(3 K_{M}+4 \mu_{M}\right)}+1\right]}$ \\
$\bar{\varepsilon}_{\mathrm{I}_{12}}^{(2)} / \varepsilon_{12}^{0}$ & $\frac{15\left(1-v_{0}\right) \mu_{M}}{\left(7-5 v_{0}\right) \mu_{M}+2\left(4-5 v_{0}\right) \mu_{I}}$ & $\frac{1}{\left.\frac{6\left(K_{M}+2 \mu_{M}\right)\left(1-f_{I}^{(2)}\right)\left(\mu_{I}-\mu_{M}\right)}{5 \mu_{M}\left(3 K_{M}+4 \mu_{M}\right)}+1\right]}$ \\
$\bar{\varepsilon}_{\mathrm{I}_{\mathrm{kk}}^{(2)}} / \varepsilon_{\mathrm{kk}}^{0}$ & $\frac{3\left(1-v_{0}\right) K_{M}}{2\left(1-2 v_{0}\right) K_{M}+\left(1+v_{0}\right) K_{I}}$ & \\
\hline
\end{tabular}

Table 2: Characteristics of the amorphous PLA films used by (Tsuji, 2002)

\begin{tabular}{l|ccccc}
\hline Specimen & $M_{n 0}\left[10^{5}(\mathrm{~g} / \mathrm{mol})\right]$ & $\left.E_{0} \mathrm{GPa}\right)$ & $X_{c 0}, X_{c}(\%)$ & $r(\mathrm{~nm})$ & Ref. \\
\hline PDLLA & 3.7 & 1.84 & 0 & 0.85 & Tsuji et al (Tsuji, 2002) \\
\hline PLLA & 5.4 & 1.83 & 0 & 1.25 & “ \\
\hline PDLA & 4.4 & 2.09 & 0 & 1.5 & “ \\
\hline PDLA/PLLA & 4.4 & 1.55 & 0 & 1 & “ \\
\hline
\end{tabular}

$M_{n 0}$ : Initial average molecular weight $E_{0}$ : Initial Young modulus

$r$ : radius of the cavities used in fitting $X_{c 0}$ : Initial degree of crystallinity

$X_{c}:$ Scission induced crystallinity 
Table 3: Characteristics of the PLLA films used for degradation experiments by different authors in the literature

\begin{tabular}{|c|c|c|c|c|c|c|c|c|c|}
\hline $\begin{array}{c}\text { Case } \\
\text { no. }\end{array}$ & Specimen & $\begin{array}{c}M_{n 0} \\
(g / m o l)\end{array}$ & $\begin{array}{c}E_{0} \\
(G P a)\end{array}$ & $\begin{array}{c}\sigma_{t} \\
(M P a)\end{array}$ & $\begin{array}{l}X_{c 0} \\
(\%)\end{array}$ & $\begin{array}{l}X_{c} \\
(\%)\end{array}$ & $\begin{array}{c}r \\
(\mathrm{~nm})\end{array}$ & $\begin{array}{c}E_{I}^{(2)} \\
(G P a)\end{array}$ & Ref. \\
\hline 1 & PLLA0 & 540000 & 1.74 & 50 & 0 & 29 & 3.75 & 15 & (Tsuji et al., 2000) \\
\hline 2 & PLLA15 & 545000 & 1.71 & 44 & 2 & 34 & 2.5 & 20 & “ \\
\hline 3 & PLLA30 & 540000 & 1.81 & 50 & 30 & 63 & 2.5 & 5.5 & “ \\
\hline 4 & PLLA45 & 525000 & 1.99 & 53 & 45 & 76 & 4 & 6 & “ \\
\hline 5 & PLLA60 & 555000 & 1.84 & 56 & 54 & 78 & 4.1 & 5.3 & “ \\
\hline 6 & PLLA100 & 555000 & 1.94 & 6.2 & 40 & 75 & 3.8 & 6.5 & (Tsuji and Ikada, 2000) \\
\hline 7 & PLLA120 & 530000 & 1.9 & 62 & 47 & 82 & 4.1 & 5.5 & “ \\
\hline 8 & PLLA140 & 550000 & 1.92 & 58 & 54 & 92 & 4.5 & 5 & “ \\
\hline 9 & PLLA160 & 390000 & 2.11 & 45 & 63 & 98 & 4.3 & 5.5 & “ \\
\hline 10 & PLLA & 158500 & 0.67 & 64.3 & 40.7 & 53.6 & 2.5 & 15 & (Weir et al., 2004) \\
\hline 11 & PLLA & 161421 & 6.9 & - & 48 & 67.3 & 3 & 20 & (Duek et al., 1999) \\
\hline 12 & PLLA & 161421 & 5.5 & - & 0 & 55.8 & 1.75 & 10 & “ \\
\hline 13 & PLLA & 42000 & 1.4 & - & 60 & 61.3 & 3.5 & 5 & (Lam et al., 1994) \\
\hline$M_{n 0}:$ & nitial numb & r average & olecular & eight & & \multicolumn{4}{|c|}{$E_{0}:$ Initial Young modulus } \\
\hline$r: \mathrm{R}$ & dius of the & avities use & in fitting & & & \multicolumn{4}{|c|}{$X_{c}:$ Scission-induced crystallinity } \\
\hline$\sigma_{t}: \mathrm{I}$ & itial tensile & strength & & & & \multicolumn{4}{|c|}{$E_{I}^{(2)}$ : Young modulus of crystals to fit the data } \\
\hline
\end{tabular}

Table 4: Characteristics of the amorphous PLA films used by (Tsuji, 2002)

\begin{tabular}{cccc}
\hline Specimen & $\begin{array}{c}\sigma_{t 0} \\
(\mathrm{MPa})\end{array}$ & $\mathrm{n}$ & $r(\mathrm{~nm})$ \\
\hline PDLLA & 40 & 1.5 & 0.85 \\
PLLA & 48 & 3.5 & 1.25 \\
PDLA & 52 & 2.5 & 1.5 \\
PDLA/PLLA & 42 & 12 & 1 \\
\hline
\end{tabular}

